\title{
Risk analysis of duo parentage testing with limited STR loci
}

\author{
M.X. Zhang1,2, H.M. Gao ${ }^{1,2}$, S.Y. Han ${ }^{1}$, Y. Liu ${ }^{1,2}$, Y.L. Tian ${ }^{1}$, S.H. Sun ${ }^{1,2}$, \\ D.J. Xiao ${ }^{1,2}$, C.T. $\mathrm{Li}^{3}$ and Y.S. Wang ${ }^{1,2}$ \\ ${ }^{1}$ Jinan Central Hospital Affiliated to Shandong University, Jinan, \\ Shandong, China \\ 2Jinan Di'en Legal Expertise Institute of Forensic Medicine, \\ Jinan Central Hospital, Jinan, Shandong, China \\ ${ }^{3}$ Institute of Forensic Sciences, Ministry of Justice, Shanghai, China \\ Corresponding author: Y.S. Wang \\ E-mail: sdjnwys@163.com
}

Genet. Mol. Res. 13 (1): 1179-1186 (2014)

Received December 18, 2012

Accepted July 15, 2013

Published February 27, 2014

DOI http://dx.doi.org/10.4238/2014.February.27.2

\begin{abstract}
The aim of this study was to evaluate whether the Goldeneye 20A system (containing 19 short tandem repeats) can avert the shortage of duo parentage tests. Among routine cases typed by the Identifiler system, we identified 42 motherless cases, 2 fatherless cases, and 34 trio cases containing 1 locus mismatch and 4 motherless cases with 2 locus mismatches. One true trio case was rejected by fatherhood testing because of the omission of the mother's genotype and because the genotype of the putative father matched that of the child. All of the cases were retyped by the Goldeneye 20A system with the mother's or father's sample. In total, 39 motherless cases were verified by one mutation, 3 motherless cases were rejected for paternity, and 4 motherless cases with 2 locus mismatches were ruled out by fatherhood testing. After adding the father's genotype, 1 motherless case was confirmed by a single-locus mutation, whereas another case was rejected by motherhood testing. The mutation and exclusion rates detected with the Goldeneye 20A system accorded with the corresponding rates identified in the Identifiler system. The trio case also rejected fatherhood without
\end{abstract}


the mother's genotype, and we found only 2 locus mismatches. Neither the Identifiler system nor the Goldeneye 20A system compensates for the absence of genetic information from the mother or father.

Key words: STR; Parentage testing; Duo case; Mutation of STR

\section{INTRODUCTION}

Short tandem repeats (STRs) exist extensively in the human genome and have been used in forensic parentage testing (Hallenberg and Morling, 2001). Mutations at STR loci have recently attracted increasing attention (Geada et al., 2001). STR mutations include two types: point mutations and replication slippages, and these STR mutations are important for studying parentage testing using forensic genetics.

DNA parentage testing is based on Mendel's laws of inheritance, which determine the way in which child's genes are inherited from its mother and father. Parentage testing can be applied to cases in which genetic information about the mother or father is missing, which are called duo cases. However, without genetic information about the mother or father, a mismatch in the STR loci in parentage testing of duo cases may cause difficulties in determining parentage. In China, approximately 80,000 civil parentage cases are tested per year, most of which are duo cases. In this study, we investigated whether the Goldeneye 20A system (composed of 19 loci) can compensate for the lack of maternal or paternal information.

\section{MATERIAL AND METHODS}

\section{Samples}

Among 2049 civil parentage tests of duo cases verified by the Identifiler system (including 1998 motherless and 51 fatherless cases), we found 42 motherless and 2 fatherless cases with 1 locus mismatch, and 4 motherless cases with 2 locus exclusions. Of the 754 trio cases typed by the Identifiler system, 34 cases were found that had 1 locus mutation. One trio case was rejected for paternity by 4 STRs, without the mother's genotype, and we observed that the STR genotype of the presumed father matched that of the child.

Blood samples were obtained from routine subjects within the Chinese Han population with their written consent. The protocol was approved by the Ethics Committee of Jinan Central Hospital, which is affiliated with the Shandong University, China.

\section{Reagents}

The Goldeneye 20A system (Peoplespot, Beijing, China; www.peoplespotinc.com) has 19 autosomal STRs and 1 sex locus, including 13 combined DNA index system (CODIS) STRs and 6 other loci (D5S818, FGA, D3S1358, TH01, D13S317, D16S539, D8S1179, D21S11, D7S820, CSF1PO, vWA, TPOX, D18S51, Penta E, Penta D, D2S1338, D19S433, D12S391, and D6S1043). The Cumulative Power of Exclusion of the system is 0.999999, and the Cumulative Power of Discrimination is 0.9999999. This system is widely used in Chinese forensic labs. 


\section{PCR and STR typing}

Genomic DNA was extracted according to the Chelex-100 protocol (Walsh et al., 1991). The Identifiler and Goldeneye 20A systems for PCR were performed according to manufacturer instructions. An ABI PRISM 310 Genetic Analyzer and the Genotyper software V3.7 were used for sequencing and genotyping, respectively.

\section{Statistical analysis}

The Cervus 2.0 statistical software was used to determine power of exclusion (Marshall et al., 1998). The paternity index (PI) was calculated when possible by using the frequencies from the Shandong Han Population in China. Parentage was calculated according to the Recommendations on Relationship Testing in China ( $\mathrm{Li}$ et al., 2010). The conclusions were classified as follows: a) parentage was rejected if the number of locus exclusion was $\geq 3$ and PI was $\leq 0.0001$; b) parentage was not rejected if the number of locus exclusion was $<3$ and was $0.0001<\mathrm{PI}<10000$; and c) parentage was confirmed if the number of locus exclusion was $<3$ and PI was $\geq 10000$.

\section{RESULTS}

\section{Mutation of STRs in duo cases verified by the Identifiler system}

When the mother's genotype was considered, only 1 locus was excluded from the analysis of 39 motherless cases (39/42), while 3 motherless cases were excluded for parentage (3/42). One rejected case consisted of samples from 2 children (Table 1). One child had 5 STRs that excluded parentage (C1: D5S818, FGA, D3S1358, D2S1338, and D19S433; PI $<0.0001)$, whereas the other child had 6 STRs excluding parentage $(C 2$ : D5S818, FGA, D3S1358, D2S1338, D21S11, and D18S51; PI <0.0001). Another rejected case that was excluded had 7 STRs (C3: FGA, TH01, D16S539, D2S1338, D21S11, CSF1PO, and TPOX; PI $<0.0001)$. Four motherless cases with 2 locus exclusions were also excluded for paternity after the mother's genotype was added (PI $<0.0001)$.

When the father's genotype was considered, 1 case excluded a maternal relationship because 4 STRs showed exclusion (C4: FGA, D3S1358, D2S1338 and D21S11; PI < 0.0001; Table 2). The other case had only 1 locus mutation.

\section{Mutation of STRs in all of the cases verified by the Goldeneye 20A system}

All of the cases were retyped with the Goldeneye 20A system. Thirty-nine motherless cases gave one locus mismatch, whereas the same 3 duo cases had more locus exclusions than were obtained with the Identifiler system $(\mathrm{C} 1$ : PI $=0.0005$; C2: PI < 0.0001; C3: PI $=0.0091$; Table 1). When maternal genotypes were considered, the same 39 duo cases had only 1 locus mutation, and the same 3 cases were also rejected on the basis of fatherhood with more STR exclusions. Locus exclusion of one fatherless case consisted of 4 STRs without the father's information $(\mathrm{C} 4$ : PI $=0.0037$; Table 2$)$, and when the father's genotype was added, 7 locus mismatches were detected (PI $<0.0001)$; another fatherless case had only 1 locus mutation. The 34 trio cases with one mismatch had only 1 mutation that was retyped by the Goldeneye 20A system. The 4 motherless cases with 2 locus mismatches gave more STR exclusions when retyped by the Goldeneye 
20A system without the mother's samples and subsequently rejected fatherhood.

\begin{tabular}{|c|c|c|c|c|c|c|c|}
\hline \multirow[t]{2}{*}{ Loci } & \multicolumn{4}{|c|}{ One duo case (including $\mathrm{C} 1, \mathrm{C} 2$ ) } & \multicolumn{3}{|c|}{ One duo case (C3) } \\
\hline & $\mathrm{AF}$ & $\mathrm{C} 1$ & $\mathrm{C} 2$ & M & $\mathrm{AF}$ & $\mathrm{C} 3$ & M \\
\hline Amelogenin & $\mathrm{X}, \mathrm{Y}$ & $\mathrm{X}, \mathrm{X}$ & $\mathrm{X}, \mathrm{Y}$ & $\mathrm{X}, \mathrm{X}$ & $\mathrm{X}, \mathrm{Y}$ & $\mathrm{X}, \mathrm{X}$ & $\mathrm{X}, \mathrm{X}$ \\
\hline D5S818 & 11,12 & 11,13 & 11,14 & 11,11 & 12,12 & 10,12 & 10,11 \\
\hline FGA & 23,24 & 19,23 & 22,23 & 23,23 & 24,25 & 22,23 & 22,26 \\
\hline D3S1358 & 15,17 & 16,17 & 16,17 & 15,17 & 15,17 & 15,16 & 15,16 \\
\hline TH01 & 7,9 & 7,9 & 7,7 & 7,9 & 7,9 & 9,10 & 9,9 \\
\hline D13S317 & 10,11 & 11,12 & 10,12 & 8,12 & 8,12 & 12,12 & 9,12 \\
\hline D16S539 & 9,13 & 9,13 & 9,9 & 9,13 & 9,11 & 11,12 & 10,11 \\
\hline D2S1338 & 20,25 & 17,19 & 18,20 & 19,20 & 23,24 & 21,24 & 17,24 \\
\hline D8S1179 & 12,16 & 12,14 & 12,12 & 12,14 & 13,15 & 11,13 & 11,15 \\
\hline D21S11 & $29,32.2$ & $29,33.2$ & $30,32.2$ & $32.2,33.2$ & 29,30 & $30,32.2$ & 30,30 \\
\hline D7S820 & 10,11 & 11,11 & 11,11 & 11,11 & 8,11 & 11,12 & 12,12 \\
\hline CSF1PO & 11,12 & 11,11 & 11,11 & 10,11 & 10,12 & 9,10 & 10,11 \\
\hline D19S433 & 14,15 & 13,15 & 14,15 & 15,15 & 14,14 & 13,14 & $13,15.2$ \\
\hline vWA & 14,16 & 14,19 & 14,19 & 17,19 & 17,18 & 14,17 & 14,16 \\
\hline TPOX & 8,8 & 8,8 & 8,8 & 8,8 & 9,11 & 8,11 & 11,11 \\
\hline D18S51 & 13,15 & 13,14 & $13.2,14$ & $13.2,14$ & 15,17 & 12,15 & 12,16 \\
\hline D6S1043 & 10,10 & 10,11 & 11,12 & 11,12 & 12,20 & 11,11 & 11,18 \\
\hline D12S391 & 16,17 & 14,15 & 13,14 & 14,16 & 18,23 & 18,20 & 20,21 \\
\hline Penta D & 8,10 & 9,12 & 10,12 & 10,12 & 12,13 & 12,13 & 9,13 \\
\hline Penta E & 11,13 & 10,11 & 10,12 & 11,12 & 17,18 & 15,21 & 15,21 \\
\hline
\end{tabular}

$\mathrm{AF}=$ alleged father $\mathrm{C} 1=$ child $1 ; \mathrm{C} 2=$ child $2 ; \mathrm{C} 3=$ child $3 ; \mathrm{M}=$ mother.

\begin{tabular}{lccc}
\multicolumn{2}{c}{ Table 2. Genotypes of one fatherless case (adding father's genotype). } & \\
\hline Loci & AM & $\mathrm{C} 4$ & $\mathrm{~F}$ \\
\hline Amelogenin & $\mathrm{X}, \mathrm{X}$ & $\mathrm{X}, \mathrm{X}$ & $\mathrm{X}, \mathrm{Y}$ \\
D5S818 & 11,13 & 10,11 & 10,12 \\
FGA & 22,24 & 18,22 & 21,22 \\
D3S1358 & 15,17 & 15,18 & 7,17 \\
TH01 & 9,9 & 7,9 & 7,8 \\
D13S317 & 10,10 & 8,10 & 9,11 \\
D16S539 & 9,9 & 9,11 & 24,25 \\
D2S1338 & 20,23 & 21,24 & 9,10 \\
D8S1179 & 12,13 & 10,13 & $31.2,31.2$ \\
D21S11 & $30,31.2$ & $28,31.2$ & 11,11 \\
D7S820 & 10,11 & 11,11 & 11,12 \\
CSF1PO & 12,14 & 11,12 & 11,13 \\
D19S433 & $14,14.2$ & 13,14 & 14,14 \\
vWA & 14,17 & 14,14 & 11,12 \\
TPOX & 8,11 & 8,11 & 16,17 \\
D18S51 & 13,14 & 14,17 & 14,16 \\
D6S1043 & 12,19 & 14,19 & 19,20 \\
D12S391 & 18,18 & 19,25 & 9,10 \\
Penta D & 9,11 & 10,10 & 13,16 \\
Penta E & 5,14 & 10,13 &
\end{tabular}

$\mathrm{AM}=$ alleged mother; $\mathrm{C}=$ child $; \mathrm{F}=$ father.

\section{Mutation analysis}

As shown in Table 3, except for the TPOX locus, 14 STRs in the Identifiler system were found to be mutated, with an average mutation rate of $1.387 \times 10^{-3}$ among the 15 STRs. The single-step mutation rate was $95.83 \%$, whereas the double-step rate was $4.17 \%$. Mutations originating from the father occurred in $83.78 \%$ of the cases, whereas maternal mutations 
were found at a frequency of $14.86 \%$, and mutations of unknown origin represented $1.36 \%$ of all cases. The ratio of mutation events in male versus female germlines was 1.6:1.

\begin{tabular}{|c|c|c|c|c|c|c|c|c|c|c|}
\hline & \multicolumn{4}{|c|}{ Duo parentage testing $(\mathrm{N}=2049)$} & \multicolumn{5}{|c|}{ Trio testing $(\mathrm{N}=754)$} & \multirow[t]{4}{*}{ Mutation rate $\left(\mathrm{x} 10^{-3}\right)$} \\
\hline & \multirow[t]{3}{*}{ No. of mutation } & \multicolumn{3}{|c|}{ Mutation origin } & \multirow[t]{3}{*}{ No. of mutation } & \multicolumn{4}{|c|}{ Mutation origin } & \\
\hline & & \multicolumn{2}{|c|}{ Father } & \multirow{2}{*}{$\frac{\text { Mother }}{1 \text { step }}$} & & \multicolumn{2}{|c|}{ Father } & \multirow{2}{*}{$\frac{\text { Mother }}{1 \text { step }}$} & \multirow{2}{*}{$\frac{\text { Without decision }}{1 \text { step }}$} & \\
\hline & & 1 step & 2 step & & & 1 step & 2 step & & & \\
\hline D5S818 & 2 & 2 & & & 1 & 1 & & & & 0.84 \\
\hline FGA & 6 & 5 & 1 & & 6 & 4 & & 2 & & 3.37 \\
\hline D3S 1358 & 2 & 2 & & & 3 & 2 & 1 & & & 1.41 \\
\hline TH01 & 3 & 3 & & & 2 & & & 2 & & 1.41 \\
\hline D13S317 & 1 & 1 & & & & & & & & 0.28 \\
\hline D16S539 & 1 & 1 & & & 3 & 3 & & & & 1.12 \\
\hline D2S1338 & 8 & 6 & 2 & & & & & & & 2.25 \\
\hline D8S1179 & 3 & 3 & & & 1 & 1 & & & & 1.12 \\
\hline D21S11 & 1 & 1 & & & 1 & 1 & & & & 0.56 \\
\hline D7S820 & 3 & 2 & & 1 & 4 & 2 & & 1 & 1 & 1.97 \\
\hline CSF1PO & 3 & 3 & & & 3 & 1 & & 2 & & 1.69 \\
\hline D19S433 & 1 & 1 & & & 4 & 1 & 1 & 2 & & 1.41 \\
\hline vWA & 4 & 4 & & & 2 & 1 & & 1 & & 1.69 \\
\hline TPOX & & & & & & & & & & 0 \\
\hline D18S51 & 2 & 2 & & & 4 & 4 & & & & 1.69 \\
\hline Total & 40 & 36 & 3 & 1 & 34 & 21 & 2 & 10 & 1 & 20.81 \\
\hline
\end{tabular}

\section{One trio case report}

One trio case was rejected for fatherhood by the Identifiler system with 4 loci excluded (PI < 0.0001; Table 4). When analyzed as a motherless case, the genotype of 15 STRs from the alleged father matched that of the child $(\mathrm{PI}=8932.8295)$. The Goldeneye 20A system identified more locus exclusions than the Identifiler system $(\mathrm{PI}<0.0001)$. An analysis of a motherless case found only 2 locus mismatches between the alleged father and child $(\mathrm{PI}=0.3109)$.

\begin{tabular}{|c|c|c|c|}
\hline Loci & $\mathrm{AF}$ & $\mathrm{C}$ & M \\
\hline Amelogenin & $\mathrm{X}, \mathrm{Y}$ & $\mathrm{X}, \mathrm{X}$ & $\mathrm{X}, \mathrm{X}$ \\
\hline D5S818 & 8,13 & 11,13 & 12,13 \\
\hline FGA & 23,23 & 23,24 & 23,24 \\
\hline D3S1358 & 15,17 & 15,17 & 15,16 \\
\hline TH01 & 7,7 & 7,9 & 7,9 \\
\hline D13S317 & 8,11 & 10,11 & 9,11 \\
\hline D16S539 & 9,10 & 9,12 & 12,12 \\
\hline D2S1338 & 18,23 & 18,24 & 18,19 \\
\hline D8S1179 & 13,16 & 15,16 & 13,16 \\
\hline D21S11 & $29,31.2$ & $29,32.2$ & $29,32.2$ \\
\hline D7S820 & 8,12 & 8,12 & 12,12 \\
\hline CSF1PO & 11,12 & 9,11 & 9,10 \\
\hline D19S433 & $14.2,15.2$ & $15.2,15.2$ & $14,15.2$ \\
\hline vWA & 14,18 & 17,18 & 17,20 \\
\hline TPOX & 8,9 & 8,9 & 8,9 \\
\hline D18S51 & 14,15 & 14,19 & 14,19 \\
\hline D6S1043 & 11,18 & 12,13 & 12,18 \\
\hline D12S391 & 19,21 & 18,21 & 21,22 \\
\hline Penta D & 9,11 & 9,12 & 9,15 \\
\hline Penta E & 15,18 & 16,16 & 16,17 \\
\hline
\end{tabular}

$\mathrm{AF}=$ alleged father; $\mathrm{C}=$ child; $\mathrm{M}=$ mother. 


\section{DISCUSSION}

In the last decade, DNA parentage tests have been widely applied to forensic cases (Budowle et al., 1999; Kupferschmid et al., 1999). Thirteen autosomal STRs of the combined DNA index system (CODIS), D5S818, FGA, D3S1358, TH01, D13S317, D16S539, D8S1179, D21S11, D7S820, CSF1PO, vWA, TPOX, and D18S51, are the basic loci of choice for forensic use. Many commercial kits are available for the robust amplification of these core STR loci and will continue to play an important role in forensics (Butler, 2006). The Identifiler system includes the 13 CODIS along with 2 additional loci, D2S1338 and D19S433, which are used in most forensic labs to determine parentage (Collins et al., 2004). It has been shown that the D6S1043 and D12S391 loci are of high value in forensic parentage testing (Tong et al., 2010), and Penta D and Penta E are often used in forensic cases as non-CODIS markers (Salem et al., 2003; Alves et al., 2005; Chouery et al., 2010).

Known as trio testing, parentage cases usually include the mother, child, and presumed father. However, forensic determination often relies on motherless or fatherless testing because of financial or other reasons. Duo case results are usually reliable without consideration of maternal or paternal data. The DNA genotypes are evaluated in motherless or fatherless cases without locus mutations. The alleged parent will be excluded when his or her genotype fails to match that of the child at a certain locus. Laboratories usually do take into consideration the circumstances of the alleged father or mother, for example, when the alleged father is a close relative of the biological father, such as a brother, father, or uncle $(\mathrm{Pu}$ and Linacre, 2007). One study has shown that 3.3\% of the genotypes in a sample of 10,000 nephew-uncle pairs completely matched when typed by the Identifiler system (Thomson et al., 2001). Therefore, evaluating these duo cases may lead to false conclusions (Brenner, 1997; Lee et al., 2000; Wenk et al., 2006). In general, investigators would consider the duo cases without any mismatches using a limited number of loci. The mutation rate of STRs is low in parentage cases (Yan et al., 2006; Lu, 2012), but the locus mutations in duo cases are important for deciding parentage (Katsumata et al., 2001).

In this study, we investigated the duo cases with one and two locus mismatches verified by the Identifiler and Goldeneye 20A systems. Using the Identifiler system, examination of the mother's genotype identified 39 motherless cases with a single STR mutation, and 3 motherless cases were excluded for parentage determination according to the guidelines (3/42; Table 1). Analysis of the father's genotype revealed that one fatherless case had one locus mutation, whereas another fatherless case was excluded by the maternal relationship (1/2; PI $<0.0001$; Table 2). The rates of mutation and exclusion from the Goldeneye 20A system were in accordance with those of the Identifiler system when considering the mother's or father's information, but the rejected cases in the Goldeneye 20A system gave more locus exclusions than in the Identifiler system. As Tables 1 and 2 show, when genotyped by the Goldeneye 20A system, the cases of $\mathrm{C} 1, \mathrm{C} 3$, and $\mathrm{C} 4$ did not reject paternal identity without the mother's information (PI: $0.0005,0.0091$, and 0.0037 ); only $\mathrm{C} 2$ rejected fatherhood (PI $<0.0001)$. If PCR amplification of STR genotypes fails multiple times, a locus mismatch is produced (Mizuno et al., 2008; Tsuji et al., 2010). We typed these cases using the Identifiler and Goldeneye 20A systems and detected no amplification failure. STR mutations usually result in 1 locus mutation in parentage testing; however, 2 locus mutations have also been found (Narkuti et al., 2007, 2008). In this study, 4 motherless cases with 2 STR exclusions were excluded for parentage because all of them had over 4 STR mismatches with the mother's STRs $(\mathrm{PI}<0.0001)$. The 
average STR mutation rate for the Identifiler system was $1.387 \times 10^{-3}$, with the single-step mutation rate being higher than the two-step rate, and the ratio of mutation events in male versus female germlines was 1.6:1 (Table 3), matching previous reports (Lu et al., 2012).

Because duo cases lack either the mother's or father's genotype, more loci are required for determining parentage (Wenk et al., 2006). In practice, one locus mismatch is considered the result of a mutation event and is therefore ignored. In this study, typing with the Identifiler system of 4 duo cases with one locus mismatch resulted in more locus exclusions than typing with the Goldeneye 20A system (4/44; Tables 1 and 2), and the mutation was a negative event. One trio case was rejected for paternity by the Identifiler system, but after omission of the mother's genotype, no mismatches were found (PI: 8932.8295). The case was retyped with the Goldeneye 20A system, without the mother's SRT genotype, and only 2 locus mismatches were detected (PI: 0.3109; Table 3); therefore, we did not rule out fatherhood. Based on these results, use of a limited number of STRs may lead to false conclusions about parentage.

The results also showed that parentage would be falsely determined in motherless or fatherless cases with limited STR loci. In terms of the ability to exclude non-parents in duo cases, neither the Identifiler system nor the Goldeneye 20A system compensates for the absence of genetic information from the mother or father.

\section{ACKNOWLEDGMENTS}

Research supported by grants from the National Nature Science Foundation, P.R. China (\#30901701 and \#81172908).

\section{REFERENCES}

Alves C, Gusmao L, Lopez-Parra AM, Soledad MM, et al. (2005). STR allelic frequencies for an African population sample (Equatorial Guinea) using AmpFISTR Identifiler and Powerplex 16 kits. Forensic Sci. Int. 148: 239-242.

Brenner CH (1997). Symbolic kinship program. Genetics 145: 535-542.

Budowle B, Moretti TR, Baumstark AL, Defenbaugh DA, et al. (1999). Population data on the thirteen CODIS core short tandem repeat loci in African Americans, U.S. Caucasians, Hispanics, Bahamians, Jamaicans, and Trinidadians. J. Forensic Sci. 44: 1277-1286.

Butler JM (2006). Genetics and genomics of core short tandem repeat loci used in human identity testing. J. Forensic Sci. 51: $253-265$.

Chouery E, Coble MD, Strouss KM, Saunier JL, et al. (2010). Population genetic data for 17 STR markers from Lebanon. Leg. Med. 12: 324-326.

Collins PJ, Hennessy LK, Leibelt CS, Roby RK, et al. (2004). Developmental validation of a single-tube amplification of the 13 CODIS STR loci, D2S1338, D19S433, and amelogenin: the AmpFISTR Identifiler PCR Amplification Kit. $J$. Forensic Sci. 49: 1265-1277.

Geada H, Ribeiro T, Brito RM, Espinheira R, et al. (2001). A STR mutation in a heteropaternal twin case. Forensic Sci. Int. 123: 239-242.

Hallenberg C and Morling N (2001). A Report of the 1997, 1998 and 1999 Paternity Testing Workshops of the English Speaking Working Group of the International Society for Forensic Genetics. Forensic Sci. Int. 116: 23-33.

Katsumata Y, Katsumata R, Yamamoto T and Tamaki K (2001). Estimating probabilities and dealing with mutations in paternity testing - verification of DNA testing with commercially available STR kits. Nihon Hoigaku Zasshi 55: 205-216.

Kupferschmid TD, Calicchio T and Budowle B (1999). Maine Caucasian population DNA database using twelve short tandem repeat loci. J. Forensic Sci. 44: 392-395.

Lee HS, Lee JW, Han GR and Hwang JJ (2000). Motherless case in paternity testing. Forensic Sci. Int. 114: 57-65.

Li L, Chengtao L, Yan L, Yuan L, et al. (2010). Recommendations on relationship testing (in China). SF/Z JD01050012010. 
Lu D, Liu Q, Wu W and Zhao H (2012). Mutation analysis of 24 short tandem repeats in Chinese Han population. Int. J. Legal Med. 126: 331-335.

Marshall TC, Slate J, Kruuk LE and Pemberton JM (1998). Statistical confidence for likelihood-based paternity inference in natural populations. Mol. Ecol. 7: 639-655.

Mizuno N, Kitayama T, Fujii K, Nakahara H, et al. (2008). A D19S433 primer binding site mutation and the frequency in Japanese of the silent allele it causes. J. Forensic Sci. 53: 1068-1073.

Narkuti V, Vellanki RN, Gandhi KP and Mangamoori LN (2007). Mother-child double incompatibility at vWA and D5S818 loci in paternity testing. Clin. Chem. Lab. Med. 45: 1288-1291.

Narkuti V, Vellanki RN, Anubrolu N, Doddapaneni KK, et al. (2008). Single and double incompatibility at vWA and D8S1179/D21S11 loci between mother and child: implications in kinship analysis. Clin. Chim. Acta 395: 162-165.

$\mathrm{Pu}$ CE and Linacre A (2007). CPI distribution and cutoff values for duo kinship testing. Chin J. Physiol. 50: 232-239.

Salem K, Yasin S, Hamad M and Jaran A (2003). Jordanian population data on five STR forensic loci: D16S539, TPOX, CSF1PO, Penta D, and Penta E. Leg. Med. 5: 251-252.

Thomson JA, Ayres KL, Pilotti V, Barrett MN, et al. (2001). Analysis of disputed single-parent/child and sibling relationships using 16 STR loci. Int. J. Legal Med. 115: 128-134.

Tong DY, Wu XY, Sun HY, Zhao H, et al. (2010). Polymorphism analysis and evaluation of nine non-CODIS STR loci in the Han population of Southern China. Ann. Hum. Biol. 37: 820-826.

Tsuji A, Ishiko A, Umehara T, Usumoto Y, et al. (2010). A silent allele in the locus D19S433 contained within the AmpFlSTR Identifiler PCR Amplification Kit. Leg. Med. 12: 94-96.

Walsh PS, Metzger DA and Higuchi R (1991). Chelex 100 as a medium for simple extraction of DNA for PCR-based typing from forensic material. Biotechniques 10: 506-513.

Wenk RE, Houtz T and Chiafari FA (2006). Maternal typing and test sufficiency in parentage analyses. Transfusion 46: 199-203.

Yan J, Liu Y, Tang H, Zhang Q, et al. (2006). Mutations at 17 STR loci in Chinese population. Forensic. Sci. Int. 162: 53-54. 